\title{
The Clinical Anatomy of the Cavernous Sinus
}

\author{
Pengfei Zhang1, Huanjiu Xi'2*, Wenhui Li \\ ${ }^{1}$ Affiliated Tiantan Hospital, Capital University of Medical Sciences, Beijing Neurosurgical Institute, Beijing, \\ China \\ ${ }^{2}$ Liaoning Medical University, Jinzhou, China \\ Email: " huanjiuxi@163.com
}

Received 18 March 2015; accepted 20 April 2015; published 21 April 2015

Copyright (C) 2015 by authors and Scientific Research Publishing Inc.

This work is licensed under the Creative Commons Attribution International License (CC BY). http://creativecommons.org/licenses/by/4.0/

(c) (i) Open Access

\begin{abstract}
Introduction: The cavernous sinus (CS) is a very important concept because it is not only interesting to anatomical theory but also useful to clinical medicine, especially in the field of surgery. This paper described the microsurgical anatomy of the CS with special attention to its concept that the CS was really venous sinus or plexus. Materials and Methods: Fifty CSs from 25 Chinese adult cadaver heads fixed in $10 \%$ methanal, whose artery and vein were injected with red and blue latex, respectively, dissected stepwise under the operating microscope. Results: Asymmetric and nonintegral blue latex distributed in the cavity of the CS to form a retina with various diameters and repeatedly diverged and converged were observed under the surgical microscope with magnification 5 - 25, after the lateral wall of the CS was opened by maxillary approach. Measurement of sinus included length, diameter and triangular structure of the CS. It is very important to understand the microsurgical anatomy of the CS for neurosurgeons. Conclusion: The CS was venous plexus rather than sinus. The lateral wall of the sinus had two layers, and the lateral cavity of the sinus really did exist even though it was very small. The triangles where maxillary approach passed were more important for neurosurgeons.
\end{abstract}

\section{Keywords}

Cavernous Sinus, Anatomy, Concept

\section{Introduction}

In 1734, in a book named “An Anatomical Exposition of the Structure of the Human Body” by James Benignus Winslow (1669-1770) began the first and only usage of the term "cavernous sinus”, thinking that it resembled

*Corresponding author. 
the cavernous body of the penis [1]. In current text, we can't find what was called the CS rather than another name. This is a very important concept because it is not only interesting to anatomical theory but also useful to clinical medicine, especially in the field of surgery. The anatomy of the CS has been studied by a number of anatomists, neurosurgeons, ophthalmologists and radiologists using gross and microscopic dissection of histological specimens and injection and corrosion [2]-[11]. However, the concept and structure of CS in anatomical literature are still controversial [12]. It was thought that CS was not an accessible area in surgery because of its depth, complex structure and impossibility in surgery [13]. The CS contained not only the venous pathway but also the cranial nerves, the sympathetic nerve and the internal carotid artery (ICA). When affected by diseases they give rise to a CS syndrome including congenital epithelial cysts, acquired infections, inflammatory disorders, vascular lesions and neoplasms [14]. The concept that the CS was sinus or plexus was related to an issue of whether an operation was possible or not in the sellar region. Neurosurgeon requires precise anatomical concept of the CS for surgical and interventional treatment. Understanding the anatomy of the CS is very important in explaining the pathology of the CS syndrome. There were many studies on structure of the CS and many articles on the complex anatomy of the CS, but measurements of triangles were seldom published in English [4]. However, relevant literature of observation to the CS by the approach through the maxillary sinus was not found. This paper is describing the microsurgical anatomy of the CS with special attention to its concept.

\section{Materials and Methods}

Fifty CS from 25 Chinese adult fresh cadaver heads were fixed in $10 \%$ methanal, whose artery and vein system were injected with red and blue latex, respectively. The branches of the vessels though smaller than $1 \mathrm{~mm}$ diameter were sufficiently filled with latex to form cast after clearing out the contents in the vessels. The specimen was opened along with $1 \mathrm{~cm}$ above the line between arch and the external occipital protuberance to remove the brain. Then it was dissected stepwise under the operating microscope. The CS with transmaxillary sinus approach combined with the frontal-temporal approach, and extradural and intradural subtemporal approach was anatomically observed using 5 - 25 magnification of the surgical microscope (LZL-11). Measurement of sinus included length, diameter and triangular structure of the CS. The area of the triangle is $A=\sqrt{[s(s-a)(s-b)(s-c)]}$ when $a, b, c$ are the length of the sides of the triangle. $s=(a+b+c) / 2$ (Heron's formula). An angle of triangle is $\sin \theta=a / c$ or $a / b$ or $b / c$ where $a, b, c$ are the length of given side and $\theta$ is the angle between $a$ and $c$ or $a$ and $b$ or $b$ and $c$.

\section{Results}

\subsection{Observation}

\subsubsection{Overview}

Asymmetric and non-integral blue latex distributed in the cavity of the CS to form a retina with various diameters and repeatedly diverged and converged were observed under the surgical microscope with magnification 5 25 after the lateral wall of the CS was opened by maxillary approach (Figure 1). There was an adjacent relationship between retina and the ICA and the structures can be separated between retina and the ICA and between structures in the retina.

\subsubsection{Superior View}

The oculomotor nerve passed through the lateral wall of the CS into the CS on top of the posterior clinoid process laterally $5.42 \mathrm{~mm}(3.73-10.50 \mathrm{~mm})$ and on free edge of the tentorium celebelum medially. The upper segment of the anterior clinoid process was found posteromedially where the optic nerve was located and distance between the points of inlet and outlet of CS was $4.77 \mathrm{~mm}(4.10-5.17 \mathrm{~mm})$. The horizontal segment of ICA in the CS, medial, lateral and poterosuperal cavity of the CS and the lateral surface of the pituitary gland showed after the dura was dissected from the point between the ICA and the oculomotor nerve backward to the posterior clinoid process (Figure 2).

\subsubsection{Lateral View}

After the removal of the lateral wall of the CS, the structure in the CS was completely revealed. It was found 


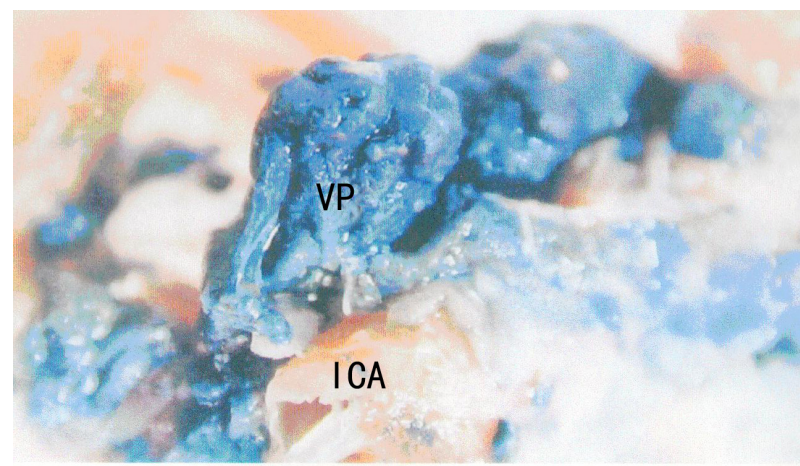

Figure 1. Venous plexus of CS. ICA: internal carotid artery; VP: venous plexus of CS. Asymmetric and non-integral blue latex distributed in the cavity of the CS to form a retina.

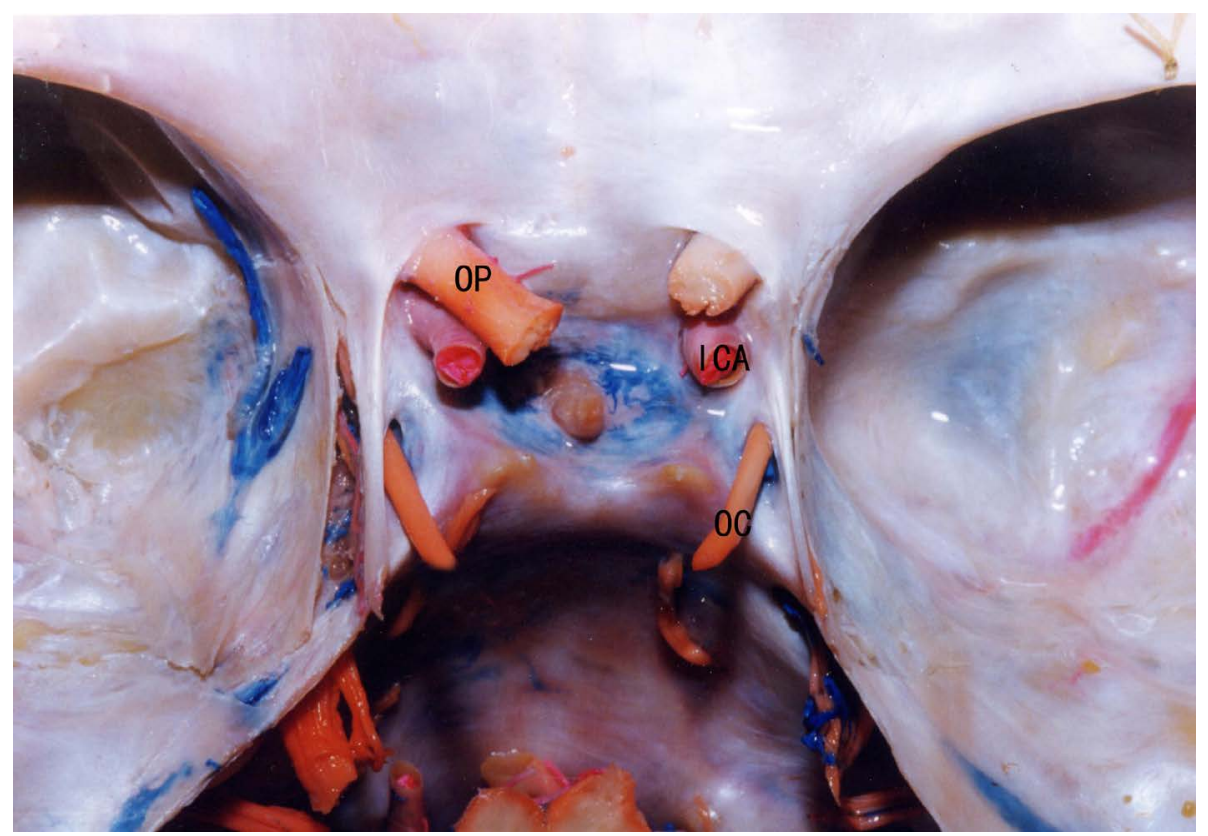

Figure 2. Superior view of CS. OP: optic nerve; ICA: internal carotid artery; OC: oculomotor nerve. CS is located on each side of sella turcica and a deep and narrow space, containing OP, ICA, OC et al.

that the oculomotor nerve entered the inferior edge of the anterior clinoid process on the lateral wall of the CS into the superior orbital fissure. The trochleal nerve running close on the free edge of the tentorium cerelelli entered CS where the oculomotor nerve passed through posterolaterally into the superior orbital fissure below the oculomotor nerve. The correlating relationship between the oculomotor nerve and the trochleal nerve was inconstant in the lateral wall of the CS, mostly aligned in company with each other and a few were hardly aligned to one another.

The abducent nerve passed through the Dorello canal to the CS and related laterally to the ophthalmic ramus of the maxillary nerve and through the superior orbital fissure to the orbital cavity. After lifting the maxillary nerve it showed that the posterior vertical segment of the ICA runs past across the ophthalmic nerve, migrated to the posterior bend segment convex, then running anteriorly to the horizontal segment, to form an anterior bend segment posterosuperally, in the end running perpendicular to the above connected anterior vertical segment outside the CS. It changes in the poterolateral direction in medial anterior clinoid process to become the superior segment of the ICA. The posterior bend segment of the ICA and the trochlear nerve running along laterally the ICA can be showed after the dura between the ophthalmic nerve and the maxillary nerve was dissected. The posterior vertical segment, bend and horizontal segment of the ICA, and the abducent nerve were seen in the 
Parkinson triangle which is composed of the trochlear nerve and the ophthalmic nerve. The horizontal and anterior bend segment can be seen along the trochlear nerve and the ophthalmic nerve forward (Figure 3).

\subsubsection{Anterior View}

The anterior bend and horizontal segment, the oculomotor nerve, the trochlear nerve, the ophthalmic nerve, the maxillary nerve, the abducent nerve, the posterosuperal and anteroinferal cavity of the CS can be showed after the anterior and inferior walls of the ICA were dissected. The anterior bend, horizontal and posterior bends segments lied in the medial-superior operating areas and were contiguous to the medial wall of the CS. Both the oculomotor and trochlear nerves were all leaning to the lateral wall of the CS. The oculomotor nerve lied on laterosuperior areas of the anterior bend segment of the ICA. The ophthalmic and the maxillary nerves lied laterally and little below the anterior bend segment. The ophthalmic nerve was on the laterosuperior of the abducent. The ICA was quite close to the abducent nerve and its part covered the oculomotor nerve. The maxillary nerve lied in inferolaterior of the anterior bend segment was close to anteroinferior wall of the CS.

\subsection{Measurement of Sinus}

\subsubsection{Length}

The intracavernous portion of the ICA was divided into the posterior vertical, the posterior bend, the horizontal, the anterior bend and the anterior vertical segments. Length of each segment of the ICA in CS can be seen in Table 1.

The distances from the oculomotor, abducent, ophthalmic nerves to the floor of ICA are shown in Table 2.

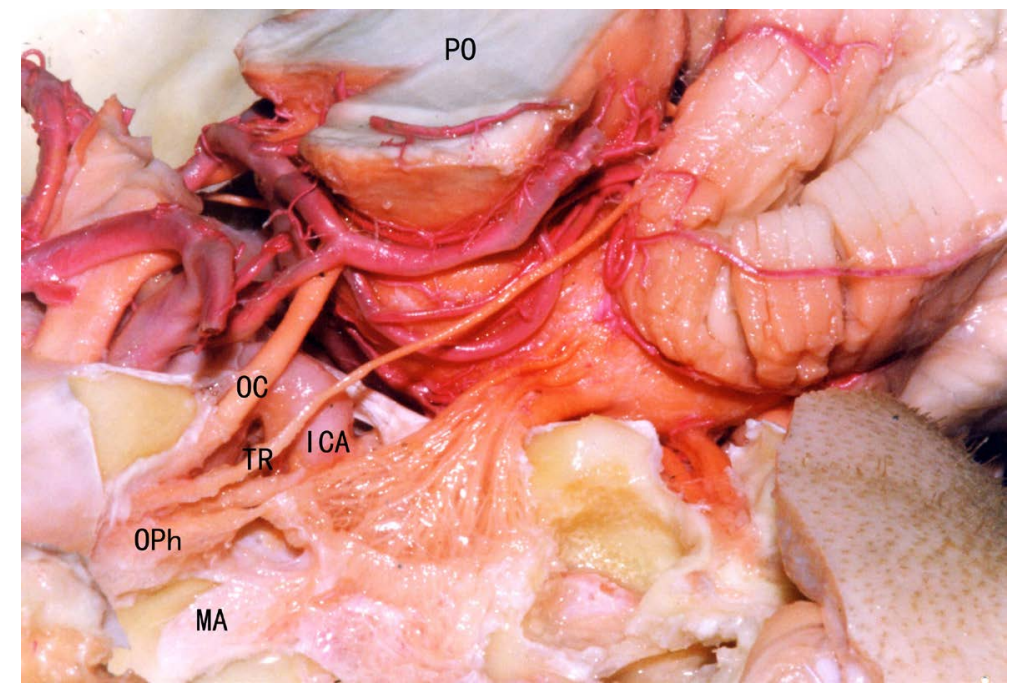

Figure 3. Lateral view of CS. TR: trochlear nerve; MA: maxillary nerve; OC: oculomotor nerve; OPH: ophthalmic nerve; PO: pons. After removal of lateral wall of CS, TR, MA, OC, OP in CS was completely revealed.

Table 1. The length of each segment of ICA in the CS $(m m, n=25)$.

\begin{tabular}{ccccc}
\hline Segment & $\mathrm{X}$ & $\mathrm{SD}$ & Min & Max \\
\hline PVS & 10.52 & 0.17 & 9.73 & 13.65 \\
PB & 3.78 & 0.22 & 2.46 & 6.12 \\
HS & 11.46 & 0.78 & 9.42 & 15.07 \\
AB & 3.32 & 1.25 & 2.41 & 6.13 \\
AVS & 5.07 & 1.34 & 4.03 & 6.72 \\
\hline
\end{tabular}

PVS: posterior vertical segment; PB: posterior bend; HS: horizontal segment; AB: anterior bend; AVS: anterior vertical segment. 
Table 2. The distances from the oculomotor, abducent, ophthalmic nerves to the floor of ICA (mm, $n=25)$.

\begin{tabular}{ccccc}
\hline & $\mathrm{X}$ & $\mathrm{SD}$ & Min & Max \\
\hline Oculomotor n. & 4.77 & 0.38 & 4.10 & 5.17 \\
Abducent n. & 3.03 & 0.35 & 2.15 & 3.88 \\
Ophthalmic n. & 1.45 & 0.63 & 0.15 & 2.76 \\
\hline
\end{tabular}

\subsubsection{Diameter}

The diameter of each segment of ICA in CS is shown in Table 3.

\subsubsection{Triangle}

The parainternal triangle: the medial and lateral borders and the base were respectively defined by the oculomotor, the trochlear nerve and the margin of tentorial dura. Parkinson triangle: the medial, lateral border and base were respectively defined by the trochlear, ophthalmic nerve and the margin of tentorial dura. The anteroexternal triangle: whose sides were the ophthalmic nerve (medial border), maxillary nerve (lateral border) and a line connecting between round foreman and the entry point of the V1 into the superior orbital fissure (base). Triangular measurement is shown in Table 4.

\section{Discussion}

\subsection{Venous Sinus or Plexus}

The concept of the CS has been debated for centuries. The hot topic has been whether the CS is the venous sinus or plexus. The CS has long been regarded as a venous sac containing the ICA before mid- $20^{\text {th }}$ century and even at first, Parkinson also favored it and explained the cause of the formation of carotid-cavernous fistula. In 1949, Taptas advanced a new concept that the CS was a venous plexus and not sinus [2]. Parkinson rediscovered that the CS was a venous plexus with venous corrosion specimens in 1973 [15]. Later, Tsuha and his co-workers discovered by X-ray that the CS consisted of complete venous passages with trabecula [16]. However, the debate of the concept of the CS is still continuing although some studies had confirmed its concept. Our study indicated that CS was not a venous sac but a venous plexus, which repeatedly diverged and converged and separated completely under the surgical microscope, and network structure closed to the ICA (Figure 1).

Our research was also confirmed by histology and imageology. Masanori et al. (2000) studied the development of the CS in fetal period and found that the CS was a collection of small venous canals with an endothelial layer, which gradually became much larger through expansion and unification. He proved that the CS is venous plexus through the development of the CS and histology [17] (Masanori et al., 2000). The concept of a venous plexus has been verified by means of contrast-enhanced, dynamic CT scanning [18].

\subsection{Wall of Sinus}

Most of the scholars thought that the CS has the superior, posterior, medial, lateral and inferior walls. The medial wall of the CS consists of a loose fibrous structure and some thought it was formed by a dural layer that constitutes the lateral wall of the sella [12] but it was questioned by Salma [19]. This also need to continue to study. The lateral wall was divided into both the internal layer which is thick and is formed by the oculomotor, trochlear nerve and ophthalmic divisions of the trigeminal never sheath and the external layer formed by the internal layer of the dura mater. And the inferior wall was formed by lateral layer of dura mater [20].

Some people thought that there was a sixth wall, that is, an anterior wall but very small corresponding to the front anterior vertical segment of ICA (namely periosteum of skull base). With two layers of the lateral wall of the CS, there were different opinions. In the past, it was thought to be only one layer, that is, the dura mater formed by the medial wall in the meddle cranial fossa. Recently, it was indicated that the lateral wall of the CS had superficial layer, that is, both the superfacial layer of the dura mater and the deep layers formed by among the nerve sheath tender of the oculomotor, trochlear nerve, the ophthalmic ramus of the trigeminal nerve and the maxillary nerve and the network memberane between sheathes. In our operating, one layer dura mater closed loosely to deep layer can be complete removed from the lateral wall of the CS when the dura mater was opened, 
Table 3. Diameter of each segment of ICA in CS $(\mathrm{mm}, \mathrm{n}=25)$.

\begin{tabular}{ccccc}
\hline Segment & $\mathrm{X}$ & SD & Min & Max \\
\hline PVS & 5.32 & 1.27 & 4.68 & 5.88 \\
PB & 5.18 & 0.73 & 4.51 & 6.10 \\
HS & 5.06 & 0.82 & 4.32 & 6.05 \\
AVS & 4.84 & 1.32 & 3.78 & 5.86 \\
AB & 4.86 & 0.32 & 4.08 & 6.72 \\
\hline
\end{tabular}

PVS: posterior vertical segment; PB: posterior bend; HS: horizontal segment; AVS: anterior vertical segment; AB: anterior bend.

Table 4. Dimensions of the triangles in the CS ( $\mathrm{mm}$, degree, $\left.\mathrm{mm}^{2}\right)$.

\begin{tabular}{cccccccc}
\hline Triangle & Medial border & Lateral border & Base & Angle (M-L) & Angle (M-B) & Angle (L-B) & Area \\
\hline Parainternal & $17.10+1.09$ & $16.33+3.02$ & $4.08+2.21$ & 13.8 & 72.3 & 93.9 & 33.24 \\
Parkinson & $16.33+3.02$ & $18.07+2.93$ & $8.04+2.34$ & 26.4 & 89.0 & 64.6 & 65.64 \\
Anteroexternal & $15.32+1.67$ & $12.34+1.71$ & $7.96+1.98$ & 31.1 & 53.3 & 95.6 & 48.88 \\
\hline
\end{tabular}

Angle (M-L): angle formed between medial and lateral borders. Angle (M-B): angle formed between medial and lateral borders; Angle (L-B): angle formed between medial and lateral borders.

which indicated the lateral wall has two layers. Our result was in keeping with Villain [21] and Campero et al. [22].

\subsection{The Cavity of the Sinus}

Harris divided the CS into the medial, anteroinferior and posteroinferior cavities according to running course [21]. The medial cavity lies between the ICA and the pituitary gland and is often closed because the ICA is twisted into the pituitary gland. The anteroinferior cavity is below the posterior bend of ICA where the abducent nerve pass. The posterosuperior cavity is posterally between the ICA and the top of the CS and sometimes closed due to artery twist. A group of scholars called the cavity between ICA and the lateral wall of the CS as the lateral cavity [20]. However, some scholars disagreed on it. The study has indicated that the lateral cavity is one between the lateral wall of both ICA in the CS and CS (Figure 4). It really did exist even though it was very small. It can be enlarged or reduced if there were some pathlogical changes.

\subsection{Nerves of Sinus}

The nerves running through or beside the lateral wall of the CS were the oculomotor, trochlear, ophthalmic, maxillary and abducent nerves. The oculomotor nerve located laterior $5.42 \mathrm{~mm}(3.73-10.52 \mathrm{~mm})$ of the posterior clinoid process. The nerve runs along the free medial edge of the tentorium celebelli and enters the top of the lateral wall of the CS into the deep layer of the anterosuperior of lateral wall of the CS to the superior orbital fissure on the inferior margin of the anterior clinoid process. It was prone to damage if the anterior clinoid process was reduced. The position of the trochlear nerve varied within wide limits and had variations on the literatures [16] [23]. We found that the trochlear nerve, running a stretch on the free edge of the tentorium cerebelli, entered the CS in the posterolaterior area where it passed through into a deep layer of the lateral wall of the CS. The running line of the oculomotor and trochlear nerve was not stable and most of specimens showed tight bundle of nerves but few did have bigger distances between the nerves. The ophthalmic and the maxillary nerves pass along the lateral wall of the CS and the ophthalmic nerve enters into the CS in the lower part of lateral wall of the CS and then moves upward obliquely through the superior orbital fissure to the orbital cavity. The abducent nerve through Dorello canal on the clivus entered the front of CS and then into the superior orbital fissure of the orbital cavity. The relationship among the oculomotor, ophthalmic, maxillary nerves and the lateral wall of CS was very precise but there were different opinions about the relationship between the trochlear nerve and the lateral wall of the CS. Thompson et al. [24] thought that the trochlear nerve lied below the lateral wall of the 


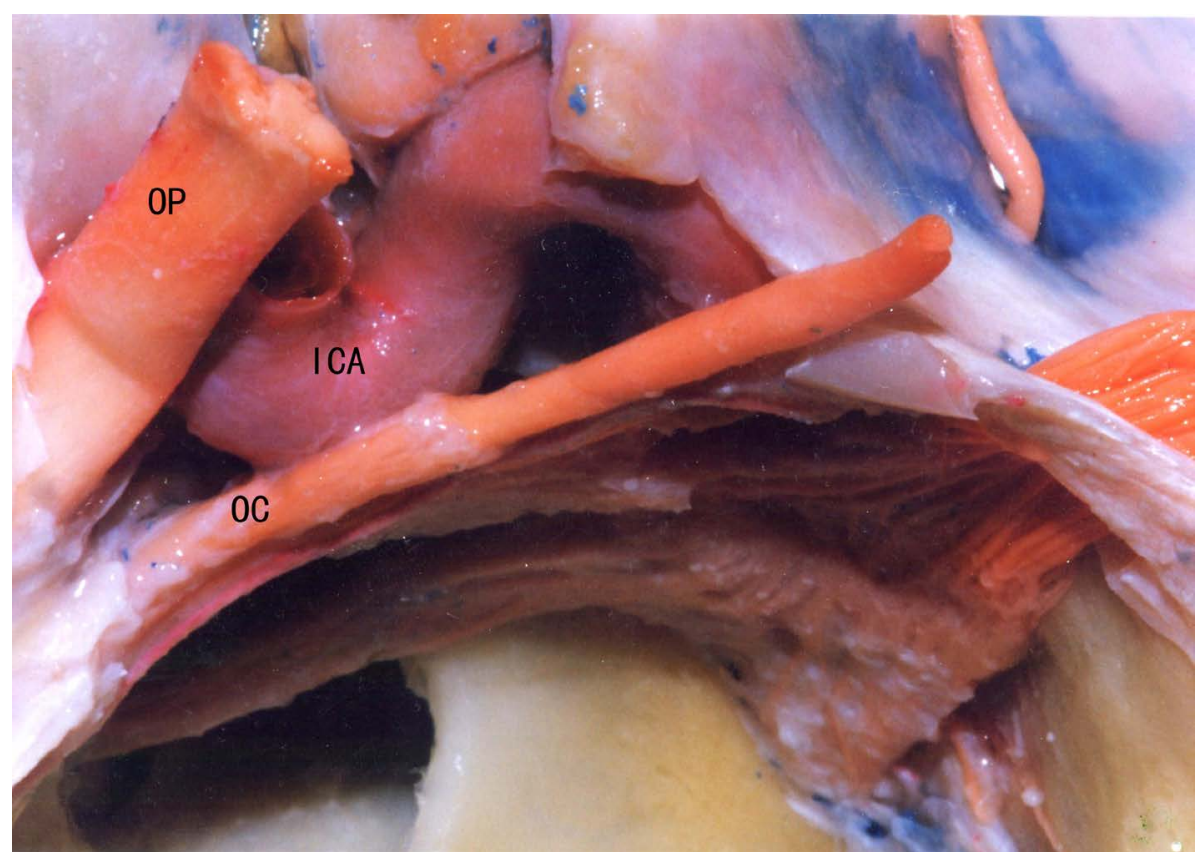

Figure 4. Cavities of CS. OP: optic nerve; OC: oculomotor nerve; ICA: internal carotid artery.

CS and passed between the lateral wall and the vessel endothelium of the CS [24]. Harris [25] indicated that the trochlear nerve passed between the superficial and the deep layers of the lateral wall and his opinion was the same as our observation. These different results may come from some specimens with incomplete deep layer and from inconsistent layer for the lateral wall, therefore, it was mistaken for the trochlear nerve running between superficial and incomplete deep layers in the deep part of the lateral wall of the CS.

\subsection{Vessels of Sinus}

The main branches of the ICA within the CS were meningohypophyseal trunk, inferior artery of the CS and the pituitary capsular artery (Mc Connell) [26] [27]. Meningohypophyseal trunk was the largest and the most constant branch of vessel mostly arising from the roof of the posterior curve of the ICA. In the report its occurrence rate was $100 \%$ and in accordance with the literature but the result in Dalgic et al. was $96 \%$ [5]. We found that the main branches of the meningohypophyseal trunk (Figure 5) were as follows:

1) The tentorial artery runs from the posterior vertical posterolaterior to the petrosal dura mater. Then it runs continuously along the free edge of the tentorium celebelli. Its branches supply the oculomotor and trochlear nerves and superior and lateral walls of the CS.

2) The dorsal meningeal artery runs from posterior bend to under the CS through the posterior wall of the CS to supply clivus dura mater and the abducent nerve.

3) The inferior hypophyseal artery runs obliquely forward from the floor of the posterior curve to supply the duta mater of the sellar floor and the dorsum sellae.

The three branches mentioned above were not all coming from the meningohypophyseal trunk and some branches came directly from the ICA. In our specimens there were 10 cases of complete type (66.7\%) and 5 cases of incomplete type (33.3\%) which were different from Dalgic (complete type, 76\%; incomplete, 24\%) (Dalgic et al., 2010). The inferior artery of the CS was mostly coming from the lateral horizontal segment of the ICA and over the abducent nerve divided into posterior and anterior branches to supply the inferior part of the lateral wall and the inferior wall of the CS, dura mater of the foramen ovale and the foramen spinosum region. The pituitary capsular artery was often absent. It was found that 2 cases of the pituitary capsular artery were from the internal face of the ICA and directly gone to anterior part of dura mater in skull base. Campero et al. [22] found that intracavernous carotid branches were the meningohypophyseal trunk (96\%), artery of the inferioe cavernous sinus (74\%), McConnell's capsular artery (10\%), carotid origin of the tentorial artery (12\%), intracavernous origin of the ophthalmic artery (2\%) [22]. 


\subsection{Triangle}

The triangles of the CS had been intensively studied since Parkinson first reported a direct surgical approach to the CS in 1965 for a carotid-cavernous fistula through triangle region formed by the oculomotor, trochlear and ophthalmic nerves and successfully treated arteriovenous fistula [3]. Rhoton thought that there were 10 triangles in and around the CS [10]. We chose important triangles for surgical purposes to measure anatomically. There were 4, 6, 2 triangles respectively on the superior, lateral wall and its neighboring areas and posterior wall of the CS (Figure 6). The angles measured in this paper were more valuable, especially that the triangles where maxillary approach passed were more important. The use of anatomical data of angles measured can direct operations relative to the CS and the main nerves and vessels were unlikely damaged when the superior, lateral and posterior walls were cut. Harris et al. [25] found the medial border averaged $13 \mathrm{~mm}$ (ranged 8 - $20 \mathrm{~mm}$ ), lateral

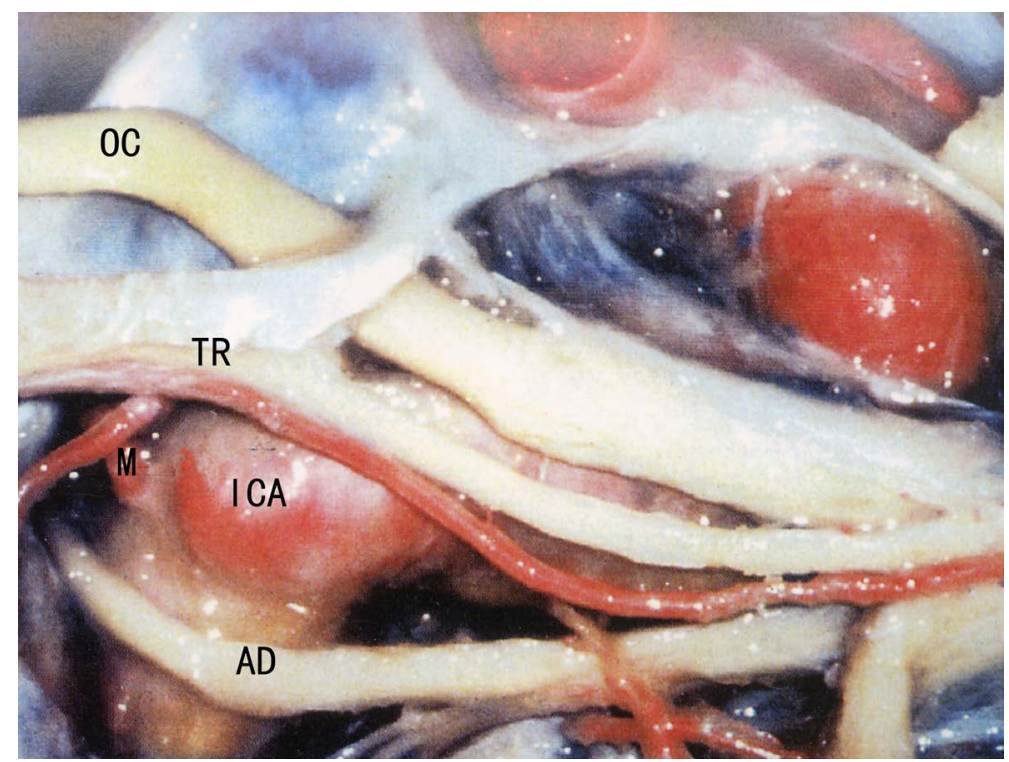

Figure 5. Meningohypophyseal artery. M: Meningohypophyseal artery; ICA: internal carotid artery; OC: oculomotor nerve. TR: trochlear nerve; AD: abducent nerve.

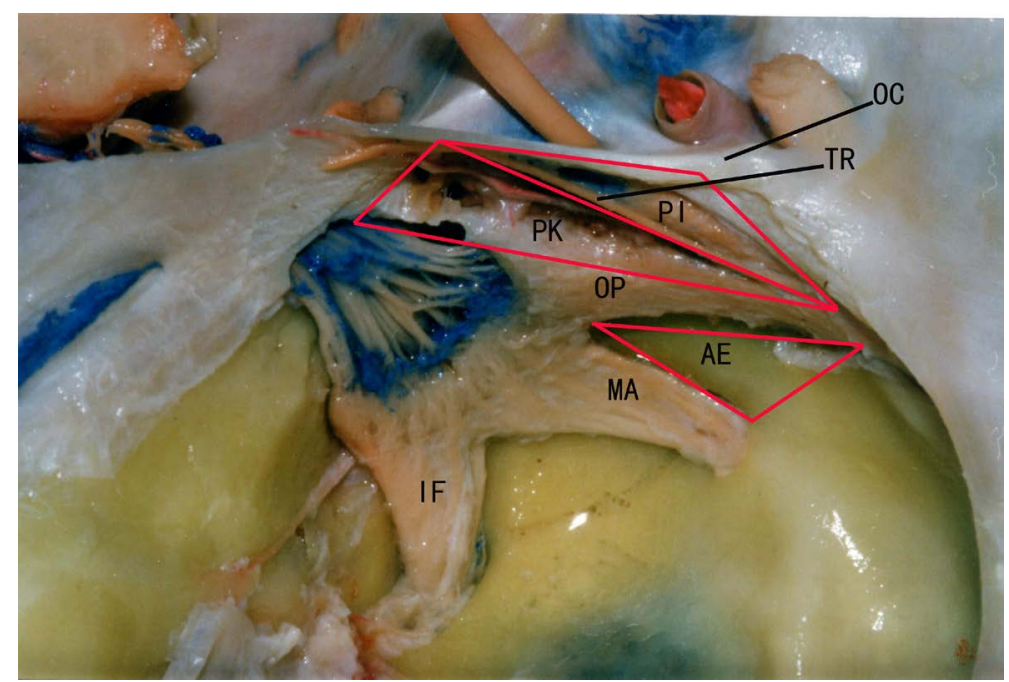

Figure 6. Triangles of CS. PI: parainternal triangle; PK: Parkinson’s triangle; AE: anterioexternal triangle; OC: oculomotor nerve; TR: trochlear nerve; MA: maxillary nerve; IF: inframandibular nerve; OP: ophthalmic nerve. 
border averaged 14 mm (ranged 5 - 24 mm), posterior border averaged 6 mm (ranged 3 - 14 mm) for Parkinson triangle. Our results differed from those of Harris et al. and some authors because of the lack of definitive standards and of methodological criteria, especially from the variations among individuals.

The lengths measured by Dalgic et al. [5] were $5 \pm 1 \mathrm{~mm}$ (ranged, 4 - $6 \mathrm{~mm}$ ), $13.5 \pm 1.5 \mathrm{~mm}$ (ranged, 12 - 15 $\mathrm{mm}$ ) and $4 \pm 1 \mathrm{~mm}$ (ranged, $3-5 \mathrm{~mm}$ ) for the posterior vertical segment, horizontal segment and the anterior vertical segment, respectively. The reasons for the differences in the results need to be studied continuously. Our data were used only for reference because of large inter-individual variations [28]. The term CS has become a barrier to understanding the structure and function of sellar region [1] and the CS is also a misnomer [4]. Therefore, it should be replaced by a new term. In the CS surgery, it is very important to expose the operating field. However, size and shape variation of the structure in the CS region are very large among individuals. Therefore, it is necessary to continue to study the CS anatomically.

\section{Support}

Supported by Grants from Beijing Newstar Plan of Science and Technology (No. 2007A042).

\section{References}

[1] Parkinson, D. (1998) Lateral Sellar Compartment O.T. (Cavernous Sinus): History, Anatomy, Terminology. Anatomical Record, 251, 486-490. http://dx.doi.org/10.1002/(SICI)1097-0185(199808)251:4<486::AID-AR7>3.0.CO;2-Q

[2] Taptas, J.N. (1982) The So Called Cavernous Sinus: A Review of the Controversy and Its Implications for Neurosurgeons. Neurosurgery, 11, 712-717. http://dx.doi.org/10.1227/00006123-198211000-00019

[3] Parkinson, D.A. (1965) Surgical Approach to the Cavernous Portion of Carotid Artery. Anatomical Studies and Case Report. Journal of Neurosurgery, 23, 474-483. http://dx.doi.org/10.3171/jns.1965.23.5.0474

[4] Isolan, G.R., Krayenbühl, N., de Oliveira, E. and Al-Mefty, O. (2007) Microsurgical Anatomy of the Cavernous Sinus: Measurements of the Triangles in and around It. Skull Base, 17, 357-367. http://dx.doi.org/10.1055/s-2007-985194

[5] Dalgic, A., Boyaci, S. and Aksoy, K. (2010) Anatomical Study of the Cavernous Sinus. Emphasizing Operative Approaches. Turkish Neurosurgery, 20, 186-204.

[6] Bedford, M.A. (1966) The “Cavernous Sinus”. British Journal of Ophthalmology, 50, 41-46. http://dx.doi.org/10.1136/bjo.50.1.41

[7] Inoune, T., Rhoton, A.L., Theele, D. and Barry, M.E. (1990) Surgical Approaches to the Cavernous Sinus: A Microsurgical Study. Neurosurgery, 26, 903-932. http://dx.doi.org/10.1227/00006123-199006000-00001

[8] McGratt, P. (1977) The Cavernous Sinus: An Anatomical Survey. Australian and New Zealand Journal of Surgery, 47, 601-613. http://dx.doi.org/10.1111/j.1445-2197.1977.tb06591.x

[9] Rhoton Jr., A.L., Hardy, D.G. and Chambers, S.M. (1979) Microsurgical Anatomy and Dissection of the Sphenoid Bone, Cavernous Sinus, and Sellar Region. Surgical Neurology, 12, 63-104.

[10] Rhoton Jr., A.L. (2002) The Supratentorial Cranial Space: Microsurgical Anatomy and Surgical Approach. Neurosurgery, 21, s375-s410. http://dx.doi.org/10.1097/00006123-200210001-00001

[11] Umansky, F., Valarezo, A. and Elidan, J. (1994) The Superior Wall of the Cavernous Sinus: A Microanatomical Study. Journal of Neurosurgery, 81, 914-920. http://dx.doi.org/10.3171/jns.1994.81.6.0914

[12] Goncalvers, M.B., do Oliveira, J.G., Williams, H.A., Alvarenga, R.M. and Landeiro, J.A. (2012) Cavernous Sinus Medial Wall: Dural or Fibrous Layer? Systematic Review of the Literature. Neurosurgical Review, 35, 147-153. http://dx.doi.org/10.1007/s10143-011-0360-3

[13] Wang, Z.M. (1994) Surgery of Skull Hase. Shanghai Science Technological Publishing House, Shanghai, 110.

[14] Ettl, A., Zwrtek, K., Daxer, A. and Salomonowitz, E. (2000) Anatomy of the Orbital Apex and Cavernous Sinus on High-Resolution Magnetic Resonance Images. Survey of Ophthalmology, 44, 303-323. http://dx.doi.org/10.1016/S0039-6257(99)00115-0

[15] Parkinson, D. (1992) Cavernous Sinus Exploration. Journal of Neurosurgery, 76, 341-342.

[16] Tsuha, M., Aoki, H. and Okamura, T. (1987) Roentgenological Investigation of Cavernous Sinus Structure with Special Reference to Paracavernous Cranial Nerves. Neuroradiology, 29, 462-467. http://dx.doi.org/10.1007/BF00341744

[17] Hashimoto, M., Yokota, A., Yamada, H. and Okudera, T. (2000) Development of the Cavernous Sinus in the Fetal Period: A Morphological Study. Neurologia Medico-Chirurgica (Tokyo), 40, 140-150. http://dx.doi.org/10.2176/nmc.40.140

[18] Bonneville, J.F., Cattin, F., Bouchareb, M. and Dynamic, C.T. (1987) Scanning of the Cavernous Sinus. In: Dolenc, 
V.V., Ed., The Cavernous Sinus, Springer-Verlag, Wien, 141-143. http://dx.doi.org/10.1007/978-3-7091-6982-7_10

[19] Salma, A. (2013) Medial Wall of the Cavernous Sinus: Dural or Fibrous Layer? Neurosurgical Review, 36, 169. http://dx.doi.org/10.1007/s10143-012-0427-9

[20] Amar, A.P. and Weisws, M.H. (2003) Pituitary Anatomy and Physiology. Neurosurgery Clinics of North America, 14, 11-23. http://dx.doi.org/10.1016/S1042-3680(02)00017-7

[21] Villain, M., Segnerbieux, F., Bonnel, F., Aubry, I. and Armand, B. (1993) The Trochlear Never: Anatomy by Microdissection. Surgical and Radiologic Anatomy, 15, 169-173. http://dx.doi.org/10.1007/BF01627696

[22] Campero, A., Campero, A.A., Martins, C., Yasuda, A. and Rhoton Jr., A.L. (2010) Surgical Anatomy of the Dural Walls of the Cavernous Sinus. Journal of Clinical Neuroscience, 17, 746-750. http://dx.doi.org/10.1016/j.jocn.2009.10.015

[23] Ziyal, I.M., Sekhar, L.N., Ozgen, T., Söylemezoğlu, F., Alper, M. and Beşer, M. (2004) The Trigeminal Nerve and Ganglion: An Anatomical, Histological, and Radiological Study Addressing Transtrigeminal Approach. Surgical Neurology, 61, 564-573. http://dx.doi.org/10.1016/j.surneu.2003.07.009

[24] Thompson, J.S. (1997) Core Textbook of Anatomy. JB. Lippincott, Philadelphia, 204-206.

[25] Harris, F.S. and Rhoton Jr., A.L. (1976) Anatomy of the Cavernous Sinus: A Microsurgical Study. Journal of Neurosurgery, 45, 169-180. http://dx.doi.org/10.3171/jns.1976.45.2.0169

[26] Rhoton Jr., A.L. (2002) The Sellar Region. Neurosurgery, 51, S1-335-S1-374. http://dx.doi.org/10.1097/00006123-200210001-00009

[27] Rhoton Jr., A.L. (2002) The Cavernous Sinus, the Cavernous Venous Plexus, and the Carotid Collar. Neurosurgery, 51, S1-375-S1-410. http://dx.doi.org/10.1097/00006123-200210001-00010

[28] Eisenberg, M.B., Al-Mefty, O., Demonte, F. and Burson, G.T. (1999) Benign Nonmeningeal Tumors of the Cavernous Sinus. Neurosurgery, 44, 949-954. http://dx.doi.org/10.1097/00006123-199905000-00008 